Bucknell University

Bucknell Digital Commons

$9-2013$

\title{
Sensory-specific Appetition: Postingestive Detection of Glucose Rapidly Promotes Continued Consumption of a Recently Encountered Flavor
}

\author{
Kevin P. Myers \\ Bucknell University, kmyers@bucknell.edu \\ Marisa S. Taddeo \\ Bucknell University \\ Emily K. Richards \\ Bucknell University
}

Follow this and additional works at: https://digitalcommons.bucknell.edu/fac_journ

Part of the Behavioral Neurobiology Commons, Behavior and Behavior Mechanisms Commons, and the Biological Psychology Commons

\section{Recommended Citation}

Myers, Kevin P.; Taddeo, Marisa S.; and Richards, Emily K.. "Sensory-specific Appetition: Postingestive Detection of Glucose Rapidly Promotes Continued Consumption of a Recently Encountered Flavor." Physiology \& Behavior (2013) : 125-133.

This Article is brought to you for free and open access by the Faculty Scholarship at Bucknell Digital Commons. It has been accepted for inclusion in Faculty Journal Articles by an authorized administrator of Bucknell Digital Commons. For more information, please contact dcadmin@bucknell.edu. 


\title{
Sensory-specific appetition: Postingestive detection of glucose rapidly promotes continued consumption of a recently encountered flavor
}

\author{
Kevin P. Myers a,b,c,*, Marisa S. Taddeo ${ }^{c}$, Emily K. Richards ${ }^{c}$ \\ a Department of Psychology, Bucknell University, Lewisburg, PA 17837, USA \\ b Program in Animal Behavior, Bucknell University, Lewisburg, PA 17837, USA \\ c Program in Neuroscience, Bucknell University, Lewisburg, PA 17837, USA
}

\section{H I G H L I G H T S}

- Intragastric glucose infused during a meal increased rats' licking and intake.

- This effect was produced by glucose but not fructose nor maltodextrin infusion.

- Intake stimulation was abolished if the flavor was switched after glucose infusion.

- Within-meal feedback from gut nutrients can alter meal size via flavor evaluation.

\section{A R T I C L E I N F O}

\section{Article history:}

Received 6 November 2012

Received in revised form 15 February 2013

Accepted 6 March 2013

\section{Keywords:}

Appetite

Satiation

Meal size

Glucose

Motivation

Flavors

Food preference

Nutrient sensing

\begin{abstract}
A B S T R A C T
It is generally thought that macronutrients stimulate intake when sensed in the mouth (e.g., sweet taste) but as food enters the GI tract its effects become inhibitory, triggering satiation processes leading to meal termination. Here we report experiments extending recent work (see Zukerman et al., 2011 [1]) showing that under some circumstances nutrients sensed in the gut produce a positive feedback effect, immediately promoting continued intake. In one experiment, rats with intragastric (IG) catheters were accustomed to consuming novel flavors in saccharin daily while receiving water infused IG $(5 \mathrm{ml} / 15 \mathrm{~min})$. The very first time glucose $(16 \% \mathrm{w} / \mathrm{w})$ was infused IG instead of water, intake accelerated within 6 min of infusion onset and total intake increased 29\% over baseline. Experiment 2 replicated this stimulatory effect with glucose infusion but not fructose nor maltodextrin. Experiment 3 showed that the immediate intake stimulation is specific to the flavor accompanying the glucose infusion. Rats were accustomed to flavored saccharin being removed and replaced with the same or a different flavor. When glucose infusion accompanied the first bottle, intake from the second bottle was stimulated only when it contained the same flavor, not when the flavor switched. Thus we confirm not only that glucose sensed postingestively can have a rapid, positive feedback effect ('appetition' as opposed to 'satiation') but that it is sensory-specific, promoting continued intake of a recently encountered flavor. This sensory-specific motivation may represent an additional psychobiological influence on meal size, and further, has implications for the mechanisms of learned flavor-nutrient associations.
\end{abstract}

(c) 2013 Elsevier Inc. All rights reserved.

\section{Introduction}

A basic heuristic principle that has emerged from a century of behavioral neuroscience research on appetite is that the meal - the "fundamental behavioral unit of eating" [2] - is largely determined by opposition between two main influences. Food palatability stimulates eating rate, whereas satiation factors that arise during eating suppress it and bring the meal to an end. Of course this basic algebra is extended and elaborated by layers of cognitive, environmental, and social influence, but at its core it is generally thought to be a system whereby

\footnotetext{
* Corresponding author at: Department of Psychology, O'Leary Center, Bucknell University, Lewisburg, PA 17837, USA. Tel.: +1 570577 3493; fax: +1 5705777007.

E-mail address: kmyers@bucknell.edu (K.P. Myers).
}

nutrients sensed in the mouth promote intake until nutrients sensed in the gut inhibit intake through negative feedback. In this paper we present experimental evidence from our lab, which supports and extends recent work from others [1], showing that this model is incomplete in including only negative-feedback immediate effects of gut nutrients. This work describes a rapidly-onsetting, positive feedback response to nutrients sensed in the gut within a meal, which stimulates ongoing intake instead of inhibiting it.

Regarding the fundamental influence of oral hedonics as a driver of intake, humans, rodents, and other omnivorous mammals clearly like the tastes of many macronutrients. The sweetness of sugars, for some species a starchy taste of complex carbohydrates, and the taste/texture of fats, are strongly hedonically positive (reviews [3-7]) The umami taste of glutamate and some ribonucleotides, which serves as a marker 
of protein content, can enhance the palatability of other food constituents, and may be perceived by some animals as palatable on its own [8-10]. These responses to a narrow category of biologically relevant stimuli serve to make nutrient-dense foods attractive and to promote intake in proportion to macronutrient content.

Were it only for these positive responses to nutrient taste, more calorically dense foods would typically be eaten in larger amounts. But this is usually not the case, especially on initial exposures, since nutrient-dense foods are also more "filling." That is, once nutrients are swallowed they begin to activate a number of satiation processes. Although the palatability of nutrient taste does passively wane within a meal due to habituation independently of postingestive feedback [11,12], to a considerable extent ongoing eating becomes actively inhibited and eventually ceases due to the afferent detection of food accumulating in the gut and the products of its digestion and absorption giving rise to negative feedback signals $[2,13]$.

The entire post-oral extent of the gastrointestinal tract is rich in receptors that transduce physical and chemical properties of ingested food. In some cases these are structurally similar to taste receptors in the mouth, possessing some of the same transduction and signaling pathways $[14,15]$. Mechano- and chemoreception of food in the gut initiates a cascade of local and systemic responses, influencing not only a variety of functions including gut motility, efficient digestion, and nutrient partitioning, but also psychological effects on the enjoyment of eating and motivation to continue. Although they are distributed and complex, the direct effects arising from nutrient stimulation of post-oral sensory pathways are thought to be exclusively inhibitory [16-19]. In fact the only known endogenous gut peptide that stimulates intake is secreted during fasting and is inhibited by ingested nutrients [20].

An important extension to this simple negative feedback model involves the well-documented effects of postingestive nutrient detection on future meal size, mediated by learning. That is, even if gut nutrient action is entirely inhibitory during a meal, animals learn about a food's postingestive nutritive actions and may preferentially select that food and eat more the next time around [21,22]. This learning is sensoryspecific, in that the oral/cephalic sensations (the particular tastes, odors, flavors, and perhaps for some species visual appearance) are remembered in association with the postingestive nutritional consequences detected in the gut. Through Pavlovian conditioning, these oral/cephalic sensations then serve as cues that elicit conditioned increases in preference and intake on subsequent encounters, so we refer to this learning as "flavor-nutrient" conditioning.

In this way animals need not rely only on the inborn repertoire of basic hedonic responses to macronutrients themselves, and can more adeptly forage in a complex environment containing a diversity of foods that could not be anticipated by genetic endowment alone, and which constantly vary in quality and availability. Learning to respond to flavors, odors and other food properties that are reliable cues for nutrient type and density becomes a predominant influence on an individual's food preferences and meal patterns. Thus the postingestive effects of nutrients come to exert a positive influence on meal size, in contrast to their immediate satiating influences. Sclafani has coined the term "appetition" (in contrast to satiation) for this category of intake-promoting effects of macronutrients sensed post-orally [23].

Much work has attempted to identify the physiological identity of the signal(s) involved in these rewarding, appetition effects, since it is still undetermined what specific receptor sites or afferent pathways are involved. One approach has been to experimentally manipulate different pathways through deafferentation, selective nutrient infusion sites, or with receptor knockout models (for overviews, see [24,25]). In sum, this work has highlighted the input of pre-absorptive nutrient detection in the proximal intestines, but another take is that it is rather difficult to completely block flavor-nutrient conditioning, suggesting that perhaps multiple, redundant afferent pathways are involved.
In our lab we have been attempting a different strategy, using behavioral methodology to investigate when during or after a meal rats show evidence of detecting nutrients and experiencing different effects on motivation. By identifying the time course of the different psychological effects of post-oral nutrient detection, we may gain some clues of the underlying physiological events.

One informative experimental paradigm we've employed (e.g., $[26,27])$ involves modifying the standard flavor-nutrient conditioning protocol. The standard protocol has rats consume non-nutritive, flavored solutions in different sessions, with distinct flavors either paired or unpaired with intragastric (IG) nutrient infusion, to study how intake is altered by the flavor-nutrient association. But in our modified protocol, instead of each training session (i.e., 'meal') consisting of one flavor, there are two flavors in consecutive sequence, with particular flavors consumed only in the beginning half of a meal and others only in the latter half. For a meal that provides appetitive postingestive stimulation with glucose, which flavor would the rats learn to prefer? If the relevant postingestive signal accrues relatively slowly and is experienced late in the meal or after, the last flavor should become most strongly associated, since Pavlovian temporal contiguity and retroactive interference effects should minimize or prevent learning about the first flavor. But for meals with IG glucose, we found this was not the case. Instead rats did learn a strong preference for the flavor encountered in the first several minutes of the meal, and in subsequent tests they only expressed that preference when hungry, like they were when they encountered it in training [26].

These experiments have led us to argue that there is an unconditioned (in the Pavlovian sense) effect of glucose detected post-orally that arises within minutes of meal initiation to support appetitive learning. This evidence for a rapid-onset US signal is consistent with a variety of evidence that the US supporting flavor-nutrient conditioning is psychologically and physiologically dissociable from the nutrient's satiating effects that terminate the meal [28], instead acting as a separate, positive influence on intake. Based on this principle, we sought to investigate the possibility that appetition does not only act to increase subsequent intake the next time the cue flavor is encountered, but may also act in an immediate, unconditioned fashion to stimulate intake within the meal as soon as beneficial nutritive effects are first detected. This proposal differs from the common view that the immediate, direct effects of nutrients in the gut are only satiating. This possibility is further suggested by the fact that strong preference for a flavor can be acquired in only a single flavor-nutrient pairing [29,30].

Such an unconditioned appetition effect has recently been reported in mouse behavior by Sclafani and co-workers [1]. In their model, IG water self-infusion was yoked to licking of flavored saccharin in an electronic esophagus preparation. Then in three consecutive 1-h sessions, a flavor was accompanied by IG glucose instead of water. As would be expected in flavor-nutrient conditioning, licking rate and total intake increased across the three flavor + glucose pairings. But the critical finding was that IG glucose increased intake within the first session, consistent with a rapid, unconditioned appetition response to gut glucose sensing.

Our experiments sought first to demonstrate this immediate appetition effect in rats. If rats rapidly detect the unconditioned appetitive effects of glucose in the gut within minutes of meal initiation, would they, upon first encounter of a novel flavor, accelerate licking and consume more of a flavor accompanied by IG glucose than when the flavor is accompanied by IG water?

But a second goal was to extend this finding by investigating its specificity. The positive effect occurring during the first flavor-nutrient pairing could reflect a nonspecific activation or arousal stemming from a rapid rise in blood glucose. But if it genuinely reflects the initial formation of a flavor-nutrient association, we could expect it to be flavor-specific, as if rapid detection of a food's nutritive consequences feeds back onto flavor evaluation 
systems to promote continued eating of that food, not just eating in general.

Our rationale for this question came from a curious observation in experiments with the consecutive flavor paradigm described previously. When the first flavor in a meal paired with glucose infusion was removed and replaced with the second flavor, rats unexpectedly tended to suppress intake of the second flavor [26]. This occurred even though the second flavor was also accompanied by IG glucose and rats could continue to self-infuse more glucose simply by continuing to consume. This pattern was not seen for a flavor switch in IG water infusion sessions, nor did this suppression indicate learned rejection of the second flavor. Instead it appeared as if the rapid-onset postingestive signal was already detected and 'attributed' to (i.e., associated with) the first flavor. That remains speculative since the experiment was not designed to examine this unexpected behavior and therefore didn't include all the control conditions needed for conclusive interpretation. Nonetheless, it did call our attention to the potential motivational significance of rapid glucose detection. Therefore we investigate in the current experiments, first, whether detecting postingestive glucose rapidly stimulates intake within a meal, and second, if that effect is specific to the flavor that accompanied onset of the glucose.

\section{Experiment 1}

The purpose of Experiment 1 was to test for an immediate appetition effect in rats. When a novel flavor is encountered for the first time and accompanied by post-oral delivery of glucose, would that stimulate intake instead of producing only the satiation effects predicted by the standard negative-feedback model? The experimental design first familiarized rats with consuming novel flavors in saccharin while being infused IG with water. The first bout of drinking in a session triggered initiation of a single, fixed rate/volume infusion ( $5 \mathrm{ml} / 15 \mathrm{~min}$ ) and thereafter rats were free to continue drinking ad lib while licking was continuously recorded and then total intake was measured. (We chose a fixed infusion triggered by meal initiation, rather than the Sclafani lab's method of animal self-driven infusion, because self-infusion links the timing and volume infusion with initial intake rate, potentially making the resulting intake curve more complicated to interpret.)

Once a stable behavioral baseline was obtained with a series of novel flavors paired with IG water, a single test session was conducted with the same protocol except that glucose was infused instead of water. In both baseline and glucose-infusion sessions, licking patterns were continuously recorded for $2 \mathrm{~h}$ (the $15 \mathrm{~min}$ during infusion and $105 \mathrm{~min}$ thereafter) on the possibility that glucose infusion might influence intake rapidly, as recently reported in mice, or instead might produce a pattern of initial suppression (satiation) followed by more rapid re-initiation of intake. This latter possibility might result if rats experienced no immediate appetition effect but began to be motivationally effected by flavor-nutrient learning sometime in the post-meal interval.

Two features of our protocol warrant explanation since they depart from previously used methods. First, we employed both food and water rationing to have rats both hungry and thirsty for testing. Postingestive conditioning studies with rodents in general (including the Sclafani lab's protocol that demonstrated immediate appetition in mice) typically use only food restriction. Our rationale for adding water restriction was to decrease the group variance in baseline saccharin intake prior to the critical data collection. In our standard procedures we typically see a wide range of saccharin acceptability in naive Sprague-Dawley rats, sometimes a bimodal distribution with a small subgroup consuming minimal saccharin when hungry but not thirsty. This would be less of a concern in prior appetition studies with C57BL/6 mice, who are more uniformly accepting of saccharin. Combined food and water restriction raises the average baseline intake and encourages a unimodal distribution. This potentially risks a ceiling that could obscure or underestimate the magnitude of an appetition effect, yet at the same time we expected it could better enable us to detect subtle biphasic effects (e.g., initial satiation followed by earlier resumption of drinking) that initially seemed potentially likely.

Second, during the initial acclimation phase prior to the critical data sessions we familiarized rats with receiving a novel flavor each day. This was intended to remove neophobia as a complicating factor. In some cases neophobia inhibits intake during the first exposure to a flavor, but more importantly, when two different novel flavors are given across days, neophobia could conceivably be sensitized or attenuated between the first and second, complicating the comparison of the two intakes. This is less of a concern in standard conditioning studies as rats ultimately receive several training exposures to each flavor. But in this study, since the critical test depends on the response to the first exposure to a novel flavor accompanied by glucose, we chose to first habituate rats to novelty per se, so that a baseline water-paired flavor and test glucose-paired flavor could each be novel yet not differentially affected by neophobia.

\subsection{Methods}

\subsubsection{Subjects}

All animal procedures were approved by the university IACUC and were consistent with the NIH Guide for the Care and Use of Laboratory Animals. Subjects were 30 experimentally naive, female SpragueDawley rats bred in our lab from stock derived from Sage Research Models (formerly Ace Animals, Boyertown, PA). Rats were approximately 170 days old and weighed $322 \pm 29.6 \mathrm{~g}$ (Mean \pm SD) prior to surgery. They were housed individually in $8 \times 16 \times 10.5^{\prime \prime}$ plastic tub cages with corncob bedding. Each rat had an intragastric (IG) Silastic catheter $(1.02 \mathrm{~mm}$ ID, $2.16 \mathrm{~mm}$ OD) surgically installed under ketamine/xylazine anesthesia (65 and $10 \mathrm{mg} / \mathrm{kg}$ ) as described in [31]. The catheter was routed from the peritoneum subcutaneously to exit between the shoulders, where it was attached to a Luer-Loc connecter that remained capped when not in use.

\subsubsection{Apparatus}

Experimental sessions were conducted in 10 identical cylindrical test chambers, $35 \mathrm{~cm}$ high $\times 25 \mathrm{~cm}$ diameter, made of opaque plastic with a wire grid floor. When placed in a test chamber the rat's IG catheter was attached to infusion tubing suspended overhead on a standard fluid swivel/counterbalance arm assembly, in turn connected to an individually computer-controlled syringe pump.

The drinking bottle was held on a motorized bottle retractor (modified Med Associates ENV-252) so that the sipper tube was accessible through an aperture at the front of the chamber. Drinking was monitored by electronic contact lickometers interfaced to a computer. This computer, which also controlled the bottle retractors, counted each rat's licks and in individually controlled the rats' IG infusion pumps. When activated, the pump delivered the IG infusion at a rate of $0.33 \mathrm{ml} / \mathrm{min}$.

\subsubsection{Procedure}

After a postoperative recovery of at least eight days, rats were adapted to a restricted feeding and drinking schedule. On this schedule rats received 14-15 g of chow in their home cages daily in the early evening, and then their drinking water was removed 30 min later (gradually reduced from $90 \mathrm{~min}$ ). A separate period of access to $.05 \%$ saccharin solution was provided in the home cage for $2 \mathrm{~h}$ daily in the mid-morning. This schedule was maintained for 10 days before the experiment proper proceeded.

Rats were then habituated to daily 2-h drinking sessions in the experimental apparatus, at the time each day they had been receiving home-cage saccharin. For the first two days rats' catheters were connected to the infusion apparatus but nothing was infused. 
Thereafter they were habituated to being infused with $5 \mathrm{ml}$ of water during the first 15 min of consuming .05\% saccharin. When a session began, once a rat's lickometer registered 20 licks its IG infusion pump was activated. This ensured the infusion did not commence until the rat had initiated a bout of sustained licking. But on this restriction schedule rats typically began drinking and activated their IG infusion within the first few seconds, and always within the first few minutes, of the 2-h session. The pump infused $5 \mathrm{ml} \mathrm{IG}$ at a constant rate of $0.33 \mathrm{ml} / \mathrm{min}$ over the next $15 \mathrm{~min}$. Thereafter, IG infusion ceased but the saccharin bottle remained available for the remainder of the session. The lickometer recorded a second-by-second record of licks throughout, and total intake from the bottle was measured by weight.

After an initial five days of habituation, the same daily protocol continued except that each day a novel flavor was added to the saccharin (McCormick brand artificial flavors, Sparks, MD; almond, banana, coconut, cinnamon, and vanilla, $0.4 \mathrm{ml}$ of extract per $100 \mathrm{ml}$ of $.05 \%$ saccharin solution). Each rat received each flavor only once across the next five days, using a balanced Latin square ordering. The purpose of this was to engage rats' attention with moderate novelty in each session, so that intake reflects an evaluative process rather than merely habitual intake of the highly familiar saccharin. In Days 1, 2, 3, and 5 of this series, the IG infusion consisted of $5 \mathrm{ml}$ of water, just as in habituation. For the critical Test, on Day 4 only, glucose (Bio-Serv, Frenchtown, NJ; concentration $16 \% \mathrm{w} / \mathrm{w}$ in tap water) was infused instead of water. For analysis purposes, this glucose-infusion Test session was compared to a Baseline created by averaging Days 3 and 5 (the water-infusion sessions immediately preceding and following the Test session). One rat was excluded from the experiment because of consistently low intakes in every session, for a final $n=29$.

\subsection{Results}

Rats consumed significantly more during the single glucose-infusion Test session than in the water-infusion Baseline. Total 2-h intakes are depicted in Fig. 1. Average Test session intake exceeded Baseline by $29 \%$ (paired $t(28)=4.3, p<.001)$.

The lick recordings allow closer inspection of how IG glucose infusion altered intake patterns within the test. The 2-h cumulative lick counts for Baseline and Test are depicted in Fig. 2 and provide a descriptive account of intake patterns. In the first several minutes Baseline and Test licking were uniformly rapid, as would be expected given the water restriction schedule. But they diverge soon thereafter, when Baseline licking begins to decelerate while Test licking remains at a higher rate for several additional minutes. Once the initial bout of drinking ceased, the records remain roughly parallel for the session's

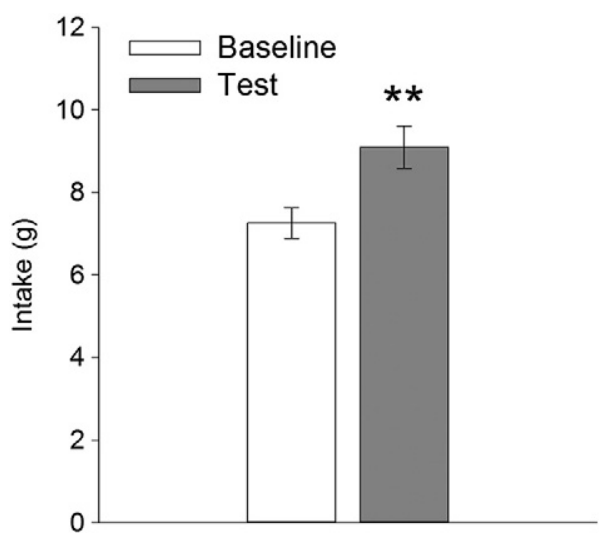

Fig. 1. Experiment 1 , Mean \pm SEM total intake of flavored saccharin in Baseline (IG water) and Test (IG 16\% glucose) sessions. In every session, $5 \mathrm{ml}$ was infused IG during the first 15 min of drinking and the total intake was measured at the end of $2 \mathrm{~h}$. Baseline is the average of two water infusion sessions on the day preceding and following the single glucose Test session. ${ }^{* *} p<.01$.
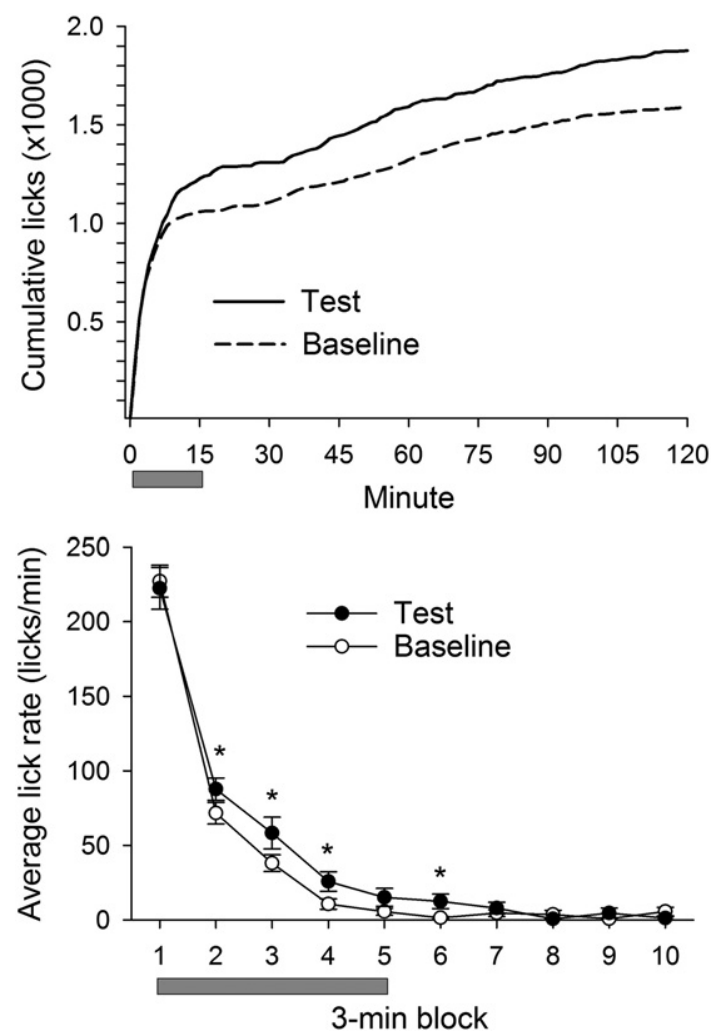

Fig. 2. Top panel: Experiment 1, cumulative lick records during the 2-h Baseline (IG water) and Test (IG 16\% glucose) sessions. Bottom panel: average (Mean \pm SEM) licking rate in each 3-min block during the initial 30 min of Baseline and Test sessions. In both panels, the gray bar under the $\mathrm{x}$-axis represents the 15 -min duration of the IG infusion. ${ }^{*} p<.05$.

remainder. Thus, the main difference was that glucose infusion caused the initial high-rate licking bout to be sustained longer.

Because of that general pattern in the cumulative records, statistical analysis focused on the initial $30 \mathrm{~min}$ (the $15 \mathrm{~min}$ during and $15 \mathrm{~min}$ immediately following the IG infusion). Lick rates (licks/min) for each minute were averaged into 10 consecutive 3 -min blocks (shown in Fig. 2, bottom) and were analyzed with a 2 (Test $v$ s. Baseline) $\times$ Time repeated measures ANOVA. While lick rates obviously decreased during the test (main effect of time block $F(9,20)=60.44, p<.0001$ ) overall licking rates were higher in Test than Baseline (main effect if session type $F(1,28)=10.98, p<.001$ ) and declined more slowly in Test than Baseline (Session $\times$ Time interaction $F(9,20)=2.51, p<.05$ ). The significant interaction was further explored with a priori planned contrasts of Baseline vs Test at each time point, which showed that Test lick rates exceeded Baseline in the second, third, fourth, and sixth 3-min block $(p ' s<.05)$

\subsection{Discussion}

Upon their first experience with IG infused glucose, hungry/ thirsty rats responded within minutes by licking at a higher rate and consuming more overall than they did during water infusion baseline tests. This is consistent with the notion that gut sensing of macronutrients, or at least glucose, may under some circumstances give rise to positive feedback effects that stimulate ongoing ingestion. This replicates the effect recently reported by Sclafani's group using a mouse model [1], even though our protocol used a different species, and different flavors, pre-testing adaptation routine, restriction schedule, and infusion volume/timing parameters. The existence of a putative immediate appetition response challenges the longstanding view that the immediate, direct effects of nutrients sensed in the gut are exclusively satiating. 
The behavioral effect we observed in this test had fairly rapid onset, being statistically detectable by the second 3-min block of the test. It is difficult to precisely pinpoint the time that glucose infusion becomes motivationally significant, since initial Baseline licking rates were quite high, presumably due to the water restriction schedule. But nonetheless, by the time licking was notably elevated, rats had received only about $30 \%$ of the $5 \mathrm{ml}$ infusion (i.e., $0.24 \mathrm{~g}$ of glucose solute).

However, before interpreting this is as an appetition effect of glucose acting as a nutrient, a chief alternative to consider is that glucose stimulated thirst. The restriction schedule made thirst a chief motivator for intake in daily sessions, with the moderately attractive taste of the flavored saccharin presumably secondary. Thus stimulation of licking by glucose infusion could instead be due to the hypertonic GI contents rapidly stimulating thirst through pre-absorptive osmoreceptors, an effect that may occur on a similar time scale [32].

To rule out a thirst effect, Experiment 2 compared effects of IG glucose and IG fructose, which have the same osmotic properties and should also stimulate licking if this was merely a thirst effect. But fructose is of special interest because it has been repeatedly shown to be ineffective at stimulating the postingestive rewarding pathways involved in flavor-nutrient learning in short sessions [33]. Since fructose only minimally supports learned positive responses, we expected it to be similarly ineffective at triggering this putative immediate appetition effect. Similarly, in an oralconsumption version of their immediate appetition protocol, Zukerman et al. [1] found that mice increased consumption when offered glucose instead of a customary sucralose, yet fructose did not produce this effect even though it is at least as sweet.

We also chose to test maltodextrin in Experiment 2, which unlike fructose is known to be effective as a postingestive stimulus for flavor-nutrient conditioning. However, we did not have a specific prediction for maltodextrin in this paradigm. On the one hand, rapid digestion of maltodextrin to glucose may allow it to produce similar rapid feedback effects. On the other hand, effects of maltodextrin have not been compared to glucose within the smaller time course relevant in this paradigm, such as in one-trial conditioning studies [29,30] or in immediate appetition studies [1]. There might be only a brief time window in the first intake bout where appetition could be behaviorally distinguished from the expected satiation effects. In any case, the main question for Experiment 2 was whether fructose would stimulate intake like glucose did in Experiment 1, since that equivalence would point towards a thirst effect rather than a true appetition effect.

\section{Experiment 2}

\subsection{Methods}

Sixteen adult female rats of similar description were used in Experiment 2. Procedures for surgery, transition to the food and water restriction schedule, and initial acclimation to saccharin drinking and IG water infusion in the test apparatus were all similar to Experiment 1, except that session length was reduced from $2 \mathrm{~h}$ to $30 \mathrm{~min}$, since in Experiment 1 Test and Baseline intake patterns only differed early in the session. In all sessions after initial familiarization, just as in Experiment 1 , IG infusion of $5 \mathrm{ml} / 15 \mathrm{~min}$ was triggered by initiation of drinking.

After rats were habituated to the daily restriction schedule and saccharin drinking with IG water infusion, the experiment transitioned to the critical data collection phase. First, each rat was familiarized in two consecutive sessions with a particular flavor added to the $.05 \%$ saccharin (either grape or berry Kool-Aid unsweetened drink mix, .05\% w/ $\mathrm{w}$ of powder added to $.05 \%$ saccharin solution), again accompanied by IG water infusion. That flavor would serve for each rat as a familiar flavor that would be given on 'washout' days intervening between each of the critical test days. On four critical test days (one Baseline and three Carbohydrate Tests), each rat received a novel Kool-Aid flavor for the first time (orange, cherry, lemon-lime, berry, grape) and the IG infusion was either water, glucose, fructose, or maltodextrin (LoDex-10; all carbohydrates from by Bio-Serv, Frenchtown, NJ). The order in which these critical tests occurred and flavor-test assignment was counterbalanced (Latin square) across rats. Carbohydrate solutions were $16 \%(\mathrm{w} / \mathrm{w})$ but infused at the same rate and volume as water infusion. In summary, Days $1,2,4,6$, and 8 were familiarization/washout days in which each rat received its familiar flavor and water infusion. Days 3, 5, 7, and 9 were critical tests in which a novel flavor appeared and was accompanied by IG infusion of water, glucose, fructose, or maltodextrin. For analysis, intake in each of the Carbohydrate Test sessions was compared to the single critical water-infusion Baseline session.

\subsection{Results}

Glucose infusion stimulated intake over Baseline by approximately $28 \%$, whereas Fructose and Maltodextrin infusions did not alter intake ( $-5 \%$ and $4 \%$ difference from Baseline, respectively). Average intakes, shown in Fig. 3, were analyzed with a repeated measures ANOVA and planned comparisons of each Carbohydrate Test to the single Baseline Test. Intake varied across test types (main effect $F(3,45)=3.25$, $p<.05$ ). But planned contrasts confirm that only the Glucose test differed from Baseline water infusion test $(p=.015)$. The Fructose and Maltodextrin tests did not differ from Water $(p=.33$ and .84$)$.

\subsection{Discussion}

This experiment replicated Experiment 1 by again demonstrating intake stimulation by IG glucose, and also found fructose and maltodextrin to be ineffective. The ineffectiveness of fructose rules out osmotic effects on thirst as the intake stimulating factor.

It was obvious, however, that neither fructose nor maltodextrin produced a satiating effect that suppressed intake relative to water infusion. This could simply reflect that baseline intake mainly represented the amount needed to satisfy thirst, and that any satiating effects of fructose or maltodextrin were neither large enough nor rapid enough to overcome that. Furthermore, tests were limited to $30 \mathrm{~min}$, so conceivably a longer test would have revealed a satiety effect on re-initiation of consumption later in time.

The effectiveness of glucose but not fructose suggests that this appetition effect may be part of the unconditioned response when nutrients stimulate peripheral afferent pathways involved in flavornutrient conditioning. Fructose infusion is generally ineffective at

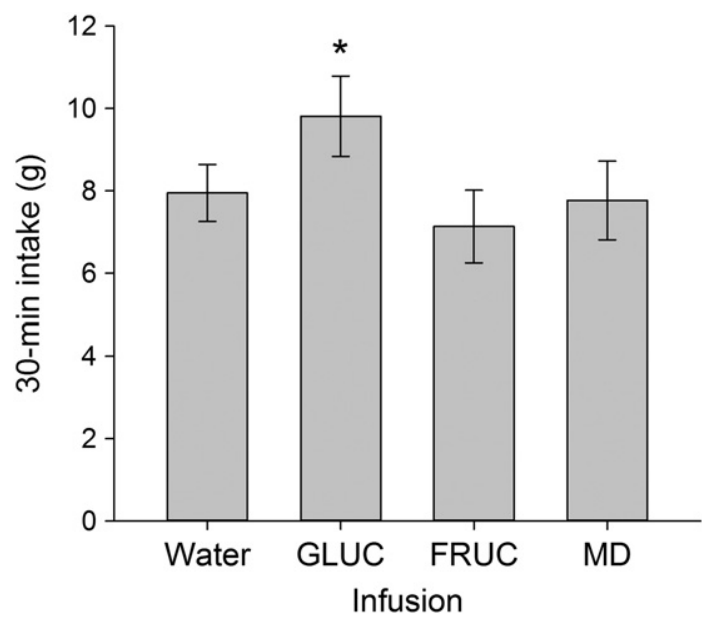

Fig. 3. Experiment 2, Mean \pm SEM total intake of flavored saccharin in 30 min sessions. In each session during the first $15 \mathrm{~min}$ of drinking rats were infused IG with $5 \mathrm{ml}$ of either water or $16 \%$ glucose, fructose, or maltodextrin. Tests were in counterbalanced order, with additional sessions of familiar saccharin flavor and water IG infusion on intervening days (not shown). ${ }^{*} p<.05$ compared to Water session. 
producing conditioned preference for a cue flavor when rats or mice are trained with several brief flavor + fructose pairings [33].

However if that is the case, it's unclear why maltodextrin did not produce an appetition effect, since it is quite effective in flavor-nutrient conditioning. One possibility is that maltodextrin (hydrolyzed to glucose) does effectively stimulate the relevant afferents for appetition, but was opposed by stimulation of other (satiating) gut sensors. But there is no evidence that maltodextrin in the gut is more satiating than glucose. Alternatively, a delay imposed by hydrolysis in the lumen, even if fairly short, could result in a different gradient of stimulation than glucose along the length of the gut lumen. The proximal intestine in particular has been implicated as a preabsorptive site in some reinforcing effects of glucose [24]. Also, as mentioned in the introduction to Experiment 1, water restriction is a somewhat unusual feature of our test protocol, and could have an effect on maltodextrin digestion that was not present in other conditioning studies. Therefore given the clear evidence of maltodextrin's postingestive reinforcing effects in several different flavor-nutrient conditioning studies, yet also the species and procedural differences between this present work and Zukerman et. al's appetition study, the status of maltodextrin in rapid positive feedback should be considered tentative and deserves additional investigation.

Of course, we have also previously argued there could potentially be several distinct nutrient-sensitive reward pathways stimulated at different time points during and after a meal that can support flavor-nutrient conditioning. Relative effectiveness of different macronutrients could reflect the subset of pathways the nutrient stimulates. The immediate appetition effect stimulated by glucose (tellingly, the single most effective nutrient molecule for conditioning preference) may represent the action of one reinforcement mechanism, which may not be necessary for all instances of flavor-nutrient conditioning. Yet Zukerman et al. [1] have also found that fat (Intralipid) stimulates immediate appetition in mice, though fat is also often found to be slower and less effective in conditioning [29,34,35]. Given the present data, we can conclude that for the simple monosaccharides glucose and fructose, their appetition effects parallel their relative effectiveness in flavor-nutrient conditioning, and this dissociation rules out thirst as an explanation for our initial results. Yet understanding the responses to different nutrients in these appetition tests will require more investigation, and it would be instructive to include a more comprehensive comparison of nutrients and non-absorbable nutrient analogs as well.

\section{Experiment 3}

Together Experiments 1 and 2 demonstrate a rapid, intake stimulating effect of gut glucose sensed within a meal. Our starting point for this analysis was an interest in isolating some of the psychobiological mechanisms involved in learned flavor-nutrient associations. Thus a main question becomes the relationship of this immediate appetition effect to those that produce conditioned effects on intake. To proceed, we sought to investigate the flavor-specificity of immediate appetition.

Learned appetition effects, whereby previous experience with a food enhances subsequent preference and intake for it, are fundamentally sensory-specific effects: the postingestive consequences specifically enhance responses to the oral sensations (e.g. flavor) that preceded them. Therefore in the next experiment we investigate whether the immediate appetition effect is also sensory specific. Is the positive feedback a generalized enhancement of ingestive motivation, or is it a specific, rapid change in evaluation of the particular flavor?

In this experiment, rats were accustomed to sessions in which they consumed a novel flavor in saccharin while receiving IG water infusion. Then the bottle would be removed and replaced with a second bottle that would alternately contain either the same or a different flavor, and they were allowed to continue drinking. Once habituated to this procedure, they were tested in sessions where glucose was sometimes infused in place of water, and while the flavor sometimes remained constant after the infusion but sometimes switched after the infusion. This allowed us to determine whether the glucose infusion stimulated a general consummatory motivation, or if it served to specifically motivate intake of the flavor that accompanied the infusion.

\subsection{Methods}

Subjects were 20 experimentally-naïve, adult female rats similar to those in previous experiments, approximately 140 days old and weighing $298 \pm 29.6 \mathrm{~g}$ (Mean \pm SD) prior to surgery. Procedures for surgery, food and water restriction, and initial familiarization to the apparatus all remained as previously described. Two rats were omitted from the experiment for consistently low or zero intake in repeated sessions, for a resultant $n=18$.

After adaptation to the daily food and water restriction schedule rats were habituated in a series of daily, 16-min long sessions in which the drinking bottle (.05\% saccharin) was inserted for $6 \mathrm{~min}$, then automatically retracted for a 2-min pause, then re-inserted for the final $8 \mathrm{~min}$. As in prior experiments, initiation of drinking triggered the onset of IG water infusion except that for this experiment the infusion rate was changed to $5 \mathrm{ml} / 7 \mathrm{~min}$ (so that the infusion would typically be completed by the end of the 2-min pause at mid-session). The purpose of this arrangement is that in eventual critical test sessions, the drinking bottle would be removed and replaced during the two-min pause so that we could observe the effect of water or glucose infusion while consuming in the first part on continued drinking from the second bottle. These session parameters were based on drinking patterns documented in Experiment 1.

Next, rats were habituated to the introduction of novel flavors in daily sessions, and to the removal/replacement of bottles during the mid-session pause. Beginning in this phase, when the bottles retracted at mid-session each rat's bottle was removed and replaced with a second, which across sessions alternately contained the same flavor as the first or switched to a new flavor. In total, fourteen different flavorings were used so that in the course of the entire experiment each rat encountered each flavor in only one session. The flavorings were McCormick extracts (almond, banana, cinnamon, coconut, coffee, hazelnut, strawberry, and vanilla, $0.4 \mathrm{ml}$ per $100 \mathrm{ml}$ of saccharin solution) and Kool-Aid unsweetened drink mixes (grape, cherry, orange, lemon-lime, black cherry, and blue raspberry, .05 g of powder per $100 \mathrm{ml}$ of saccharin solution). This procedure continued daily for six habituation sessions (three same, three switch) before the experiment proceeded.

Finally, four critical test sessions were conducted. Just as in the habituation sessions, these alternated so that in half the sessions the flavor stayed the same at mid-session and in half it switched at mid-session. In two test sessions the IG infusion was water as it always had been, but in two sessions glucose $(16 \% \mathrm{w} / \mathrm{w})$ was infused instead. Thus there was one critical session of each combination: water vs. glucose infusion, and same vs. switched flavor. The order of the four types of tests and the flavors used within each type of test varied across rats. Intakes from the first and the second bottle in each session were measured by weight.

The results of our previous experiments would predict intake stimulation by glucose infusion. But the critical comparison involves intake during the last part of each session (after the bottles are replaced) to determine whether that stimulation by glucose is specific to the flavor that accompanied it. In other words, would enhanced intake from the second bottle depend on the flavor remaining the same? Comparing second-bottle intake when water was infused indicates any effect of flavor switching per se. If the rapid positive feedback effect of glucose is a non-specific enhancement of general ingestive motivation, then intakes from the second bottle should be greater in glucose-infused than water-infused tests regardless of whether the second bottle contained the same or different flavor as the first. Yet if, as we hypothesized, 


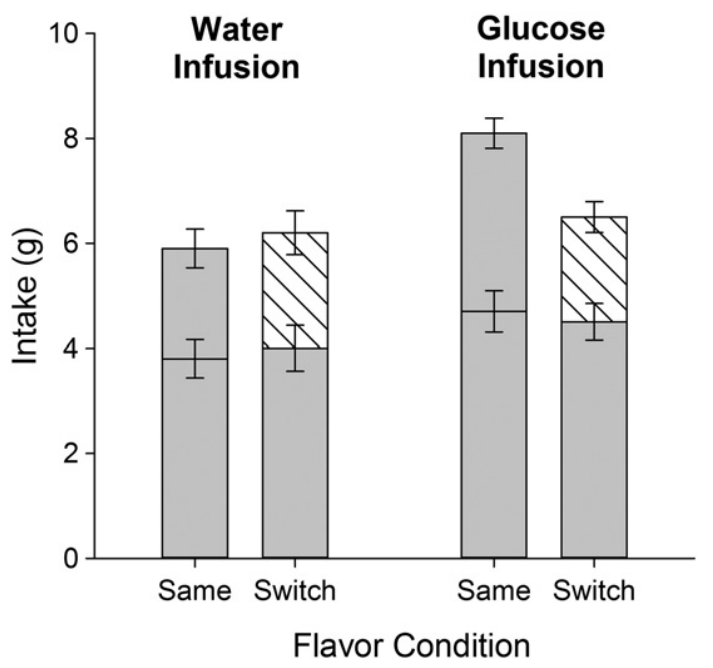

Fig. 4. Mean \pm SEM intakes in the critical test sessions of Experiment 3. In these sessions a bottle of flavored saccharin was removed after $6 \mathrm{~min}$ and replaced with a second bottle that contained the same or a different flavor for an additional $8 \mathrm{~min}$. The bottom portion of the bar represents intake from the first bottle and the top portion of the bar representing intake from the second bottle. When the entire bar is gray, the second bottle in the session contained the same flavor as the first bottle. When the top portion is hatched, the flavor was switched. The two bars on the left are tests with IG water infusion, and the two bars on the right are tests with IG glucose.

glucose infusion specifically promotes intake of a recently encountered flavor, intake stimulation in the glucose tests should only be seen when the second bottle contained the same flavor as the first.

\subsection{Results}

Glucose infusion during the first part of the test significantly stimulated intake in the latter portion of the test only when the second bottle contained the same flavor as the first bottle. This pattern is clearly shown in Fig. 4, which depicts average intakes from the first bottle (initial $6 \mathrm{~min}$ ) and second bottle (final $8 \mathrm{~min}$ ) in each type of test.

In the initial $6 \mathrm{~min}$, intakes were approximately equivalent in all tests, according to a 2 (glucose $v s$. water infusion) $\times 2$ (same $v$ s. switch condition) ANOVA on first bottle intakes. Rats did consume marginally more in this first part of the two glucose infusion sessions than in water sessions, perhaps reflecting the initial onset of the postingestive stimulatory effect, but that difference was short of statistical significance (main effect of infusion type, $F(1,17)=3.68, p=.063$ ). There was clearly no effect of the same $v s$. switch manipulation in first bottle intakes, nor did this interact with infusion type, $F^{\prime} s(1,17)=0.02$ and 0.18 , respectively, $p ' s>.05$, which is precisely as expected since the same/switch manipulation followed this measurement in time.

Intake from the second bottles depended on an interaction between infusion type and the flavor manipulation. In a 2 (glucose vs. water infusion) $\times 2$ (same $v$ s. switched flavor condition) ANOVA on second bottle intakes, the critical result is the significant interaction between the two main variables, $F(1,17)=4.83, p<.05$. Second bottle intake was higher for glucose infusion than water infusion only in the same-flavor test. But glucose infusion was ineffective at stimulating intake when the flavor switched, so second bottle intake resembled water infusion. Thus there was no overall main effect of glucose infusion nor the switch manipulation on their own, F's (1, $17)=1.93$ and 2.64, respectively, $p$ 's $>.05$.

A series of a priori planned contrasts confirm these results. In direct comparisons, second-bottle intake in the glucose-same test exceeded intake in the water-same test as well as in the glucose-switch test $(p ' s<.05)$. Second-bottle intake in water-switch, water-same, and glucose-switch were all similar to each other (lowest pairwise $p=$ $0.63)$.

\subsection{Discussion}

This experiment revealed that intake stimulation by post-oral glucose is sensory-specific. The glucose infusion delivered in the first part of the session promoted continued intake in the second part only when the flavor remained the same. When the flavor that accompanied glucose infusion in the first part was replaced with a different flavor, consumption was not elevated, and rats treated this second flavor no differently than when they had been infused with water. Clearly, the failure of glucose to stimulate intake of the switched flavor was not due to a general aversive reaction to the flavor switching procedure or sudden appearance of a novel flavor mid-meal, since in water-infusion sessions rats were apparently indifferent to the flavor switch.

This finding further adds to the evidence that initial detection of postingestive nutrient consequences (at least with glucose) can positively affect meal size, but moreover supports our prior, more speculative suggestion [26] that gut glucose onset is 'attributed' to the flavor being consumed. Considering this as a positive-feedback influence on meal intake, it appears that the feedback specifically works through flavor evaluation mechanisms. That doesn't necessarily mean the flavor becomes 'more palatable' since incentive salience can be affected separately from hedonics. But it does point to the involvement of oral-sensory evaluation, rather than more generalized influences on intake.

We and others have recently shown flavor-nutrient associations sufficient to alter future food selection can be acquired from a single meal $[29,30]$. Here it seems that those associations are perhaps being formed within the first several minutes of that single experience, based on the rapid unconditioned effect of gut glucose specifically enhancing the flavor that accompanied its onset.

\section{Discussion and conclusions}

The positive, intake-stimulating effects of nutrients tasted in the mouth and the inhibitory, negative-feedback effects of nutrients in the gut are widely recognized as two main psychobiological influences shaping meal size. As evidence has gradually accumulated that postingestive effects of nutrients also exert positive (albeit delayed) 'appetition' effects on subsequent meal size through learning, this was easily reconciled with the same basic model. In learned appetition, postingestive detection of glucose or other macronutrients at Time 1 exert an influence on preference and meal size at Time 2 because learning alters the reaction to oral-cephalic stimulation. That is, the taste or flavor of a food can become regarded as more palatable, or, through incentive sensitization mechanisms might be more accurately described as becoming more 'interesting' or 'attention-getting' [36-38]. In any case the learned appetition effect based on prior flavor-nutrient learning is still viewed as a conditioned response to oral-cephalic sensations.

Indeed there are many other examples of ingestive motivation, intake rate, physiological or subjective appetite measures being stimulated through positive feedback elicited by initial oral-cephalic contact with food (e.g., [39-47]). Commonly called an "appetizer effect," the first few bites of a palatable food can stimulate interest and motivate continued eating. But these responses do not involve the immediate feedback from postingestive stimuli. Even when they do involve postingestive input, they are still typically viewed as responses to the taste, odor, or other sensory properties of food, mediated by learning from prior experience.

What sets apart the immediate appetition effect described here from these other positive feedback influences is that it was a direct response to post-oral nutrient detection per se that immediately enhanced intake. The evidence for learned appetition effects did not, until recently, require questioning the view that the immediate, direct effects of nutrients acting post-orally within a meal are exclusively inhibitory within that meal. The evidence is now accruing that 
the basic positive (taste) $v$ s. negative (satiation) model must account for a rapid positive feedback from the gut.

Our results depict an unlearned positive feedback response to gut nutrient detection that replicates the recent report of Zukerman, Ackroff \& Sclafani using mice [1]. We found similar effects of glucose on licking within the first test session, despite several notable differences in test protocol. The Zukerman et al. study used no water restriction and a lower saccharin vehicle concentration, so that a ceiling effect on baseline licking would not obscure a positive effect. We tested rats on a stringent food and water restriction schedule that together with the moderate saccharin vehicle engendered higher initial lick rates. We chose this approach expecting we might see initial suppression of intake followed by quicker re-initiation. The two sets of results show intake stimulation by post-oral glucose can occur with either low or high initial motivation. Our method also differed by employing a fixed, bolus infusion rather than a self-controlled infusion yoked to ongoing drinking. The latter method may more realistically mimic natural eating, but for this initial investigation we chose to standardize the IG "dose" to simplify interpretation of the cumulative intake curve. With self-controlled infusion, at each time point different experimental subjects have received a different amount of glucose, and low initial drinking rate could yield a smaller, potentially ineffective IG infusion. Finding qualitatively similar effects despite these procedural differences highlights the significance of rapid nutrient sensing in the initial minutes of a meal on influencing total intake.

It is worth considering why the existence of this positive feedback effect had gone undocumented until fairly recently. There is a wealth of research on the afferent detection and physiological and behavioral effects of gut nutrients, with many reports clearly showing intake suppression by nutrients detected in the gut after oral consumption, or after intragastric or intraintestinal infusion. One reason may be that the guiding theoretical emphasis on negative feedback effects has led to test protocols developed with time and treatment parameters that are most sensitive to satiation effects. The positive feedback effect we have measured onsets rapidly, and just like the innate intake-promoting effects of sweet taste, it would lead to more rapid accumulation of food in the stomach. Careful behavioral observation of meal microstructure may be useful for dissociating opposing positive and negative feedback mechanisms that would ultimately tend to obscure the other's net contribution to meal size [48].

But we also propose another psychological factor that may be critically important: flavor novelty during the immediate appetition test. In our protocol and in Zukerman et al., the initial test with IG glucose infusion was also the subjects' first encounter with that flavor. That design stems from the fact that the research was initially concerned with how rodents acquire flavor-nutrient associations. In our protocol appetition is evident when licking and intake of the novel flavor accompanied by glucose are compared to baseline tests with equally novel flavors. Thus stimulation is not a response to novelty per se, but may be promoted by (or may in fact require) flavor novelty when postingestive glucose is detected. In fact, an additional unpublished experiment is the only one from our lab so far that failed to produce immediate appetition, and it involved IG glucose tested with a familiar flavor instead of a novel one. Thus the historical tendency of experiments to reveal satiating rather than appetitive effects of gut nutrient detection could also reflect that those experiments typically involve consumption of a familiar test substance (e.g., chow or sucrose solution for rats, a common test food for humans).

The putative influence of flavor novelty is consistent with another interesting psychological property revealed by our experiments: the immediate appetition effect is sensory-specific. Thus the central network that integrates the positive gut feedback appears to operate on sensory-evaluative processing of oral input. This is informative especially since it will be interesting to determine the relationship between the (apparently unlearned) responses to gut nutrient detection and the better-known learned acceptance and preference effects produced by flavor-nutrient conditioning. It has been repeatedly demonstrated in several experimental paradigms that pairing a specific flavor with positive nutritive consequences detected post-orally creates sensory-specific associations that influence future food selection and intake. The specific flavor not only becomes more strongly preferred, in many cases but it also becomes more hedonically positive and consumed in increasingly larger amounts. Does the intake enhancement seen in the current experiments represent the initial expression of that learned change?

Two other recent findings are directly relevant to that question. First, conditioned flavor preference requires only minimal experience, and can result from only a single flavor + nutrient pairing comparable to a single, small meal $[29,30]$. Second, in conditioned preference, some of the unconditioned rewarding effects of glucose appear to onset fairly rapidly to produce preference for flavors eaten at the beginning of the meal [26]. Taken together with this new finding that the immediate, within-meal stimulation by gut glucose sensing is flavor-specific makes it all the more likely that this represents rapid initial acquisition of flavor-nutrient associative learning. However we reiterate our previous point that there may prove to be several different afferent pathways sensitive to nutrient consequences that act independently as unconditioned stimuli in conditioning different psychological/behavioral components of ingestion, with these early-meal effects being only one. We failed to find an effect of maltodextrin in this test, although maltodextrin is clearly effective in flavor-nutrient conditioning, so this rapid onset effect may be sufficient but not necessary. Thus additional work can focus on which gut receptor and afferent pathways play roles in the time course immediate positive and negative feedbacks within a meal, and in plasticity of appetite and satiety mechanisms across meals.

\section{References}

[1] Zukerman S, Ackroff K, Sclafani A. Rapid post-oral stimulation of intake and flavor conditioning by glucose and fat in the mouse. Am J Physiol Regul Integr Comp Physiol 2011;301:R1635-47.

[2] Smith GP. The direct and indirect controls of meal size. Neurosci Biobehav Rev 1996;20:41-6.

[3] Berridge K. Measuring hedonic impact in animals and infants: microstructure of affective taste reactivity patterns. Neurosci Biobehav Rev 2000;24:173-98.

[4] Drewnowski A. Taste preferences and food intake. Annu Rev Nutr 1997;17:237-53.

[5] Sclafani A. Starch and sugar tastes in rodents: an update. Brain Res Bull 1991;27: 383-6.

[6] Steiner JE, Glaser D, Hawilo ME, Berridge KC. Comparative expression of hedonic impact: affective reactions to taste by human infants and other primates. Neurosci Biobehav Rev 2001;25:53-74.

[7] Warwick ZS, Schiffman SS. Role of dietary fat in calorie intake and weight gain Neurosci Biobehav Rev 1992;16:585-96.

[8] Bachmanov AA, Tordoff MG, Beauchamp GK. Intake of umami-tasting solutions by mice: a genetic analysis. J Nutr 2000;130:935S-41S.

[9] Beauchamp GK. Sensory and receptor responses to umami: an overview of pioneering work. Am J Clin Nutr 2009;90:723S-7S.

[10] Yamaguchi S, Ninomiya K. Umami and food palatability. J Nutr 2000;130:921S-6S.

[11] Swithers SE, Hall WG. Does oral experience terminate ingestion? Appetite 1994;23:113-38.

[12] Epstein LH, Temple JL, Roemmich JN, Bouton ME. Habituation as a determinant of human food intake. Psychol Rev 2009;116:384-407.

[13] Smith GP. The controls of eating: a shift from nutritional homeostasis to behavioral neuroscience. Nutrition 2000;16:814-20.

[14] Iwatsuki K, Uneyama H. Sense of taste in the gastrointestinal tract. J Pharmacol Sci 2012;118:123-8.

[15] Egan JM, Margolskee RF. Taste cells of the gut and gastrointestinal chemosensation. Mol Interv 2008;8:78-81.

[16] Bray GA. Afferent signals regulating food intake. Proc Nutr Soc 2000;59:373-84.

[17] Cummings DE, Overduin J. Gastrointestinal regulation of food intake. J Clin Invest 2007; 117:13-23.

[18] French SJ, Cecil JE. Oral, gastric and intestinal influences on human feeding. Physiol Behav 2001;74:729-34.

[19] Ritter RC. Gastrointestinal mechanisms of satiation for food. Physiol Behav 2004;81:249-73.

[20] Gomez G, Englander EW, Greeley GH. Nutrient inhibition of ghrelin secretion in the fasted rat. Regul Pept 2004;117:33-6.

[21] Sclafani A. Macronutrient-conditioned flavor preferences. In: Berthoud H, Seeley $\mathrm{R}$, editors. Neural and metabolic control of macronutrient intake. Boca Raton, FL: CRC Press; 1999. p. 93-107. 
[22] Sclafani A. Post-ingestive positive controls of ingestive behavior. Appetite 2001;36: 79-83.

[23] Sclafani A. Gut -brain nutrient signaling. Appetition vs. satiation. Appetite 2012, http://dx.doi.org/10.1016/j.appet.2012.05.024.

[24] Ackroff K, Yiin YM, Sclafani A. Post-oral infusion sites that support glucose-conditioned flavor preferences in rats. Physiol Behav 2010;99:402-11.

[25] Sclafani A, Ackroff K. Role of gut nutrient sensing in stimulating appetite and conditioning food preferences. Am J Physiol Regul Integr Comp Physiol 2012;302: R1119-33.

[26] Myers KP, Whitney MC. Rats' learned preferences for flavors encountered early or late in a meal paired with the postingestive effects of glucose. Physiol Behav 2010;102:466-74.

[27] Myers KP. Rats acquire stronger preference for flavors consumed towards the end of a high-fat meal. Physiol Behav 2013;111-112:179-89.

[28] Sclafani A, Ackroff K. The relationship between food reward and satiation revisited. Physiol Behav 2004;82:89-95.

[29] Ackroff K, Dym C, Yiin YM, Sclafani A. Rapid acquisition of conditioned flavor preferences in rats. Physiol Behav 2009;97:406-13.

[30] Myers KP. Robust preference for a flavor paired with intragastric glucose acquired in a single trial. Appetite 2007;48:123-7.

[31] Davis JD, Campbell CS. Chronic intrajugular, intraportal, gastric, and duodenal cannulae for the rat. In: Singh D, Avery DD, editors. Physiological techniques in behavioral research. Monterey, CA: Brooks Cole; 1975. p. 163-77.

[32] Stricker EM, Hoffmann ML. Presystemic signals in the control of thirst, salt appetite, and vasopressin secretion. Physiol Behav 2007;91:404-12.

[33] Ackroff K. Learned flavor preferences. The variable potency of post-oral nutrient reinforcers. Appetite 2008;51:743-6.

[34] Lucas F, Sclafani A. Differential reinforcing and satiating effects of intragastric fat and carbohydrate infusions in rats. Physiol Behav 1999;66:381-8.

[35] Revelle CH, Warwick ZS. Flavor-nutrient learning is less rapid with fat than with carbohydrate in rats. Physiol Behav 2009;97:381-4.
[36] Myers KP, Sclafani A. Conditioned enhancement of flavor evaluation reinforced by intragastric glucose: I. Intake acceptance and preference analysis. Physiol Behav 2001;74:481-93.

[37] Myers KP, Sclafani A. Conditioned enhancement of flavor evaluation reinforced by intragastric glucose. II. Taste reactivity analysis. Physiol Behav 2001;74:495-505.

[38] Myers KP, Sclafani A. Conditioned acceptance and preference but not altered taste reactivity responses to bitter and sour flavors paired with intragastric glucose infusion. Physiol Behav 2003;78:173-83.

[39] Cornell CE, Rodin J, Weingarten H. Stimulus-induced eating when satiated. Physiol Behav 1989;45:695-704.

[40] Fedoroff IC, Polivy J, Herman CP. The effect of pre-exposure to food cues on the eating behavior of restrained and unrestrained eaters. Appetite 1997;28:33-47.

[41] Lambert KG, Neal T, Noyes J, Parker C, Worrel P. Food-related stimuli increase desire to eat in hungry and satiated human subjects. Curr Psychol 1991;10:297-303.

[42] Epstein LH, Rodefer JS, Wisniewski L, Caggiula AR. Habituation and dishabituation of human salivary response. Physiol Behav 1992;51:945-50.

[43] Hill AJ, Magson LD, Blundell JE. Hunger and palatability: tracking ratings of subjective experience before, during and after the consumption of preferred and less preferred food. Appetite 1984;5:361-71.

[44] Myers KP, Arnold HM, Hall WG. Sensitization of ingestive responding in preweanling rats: time course and specificity. Behav Neurosci 1997;111:413-23.

[45] Swithers SE, Martinson FA. Habituation of oral responding in adult rats. Behav Neurosci 1998;112:213.

[46] Terry WS. Effects of food priming on instrumental acquisition and performance. Learn Motiv 1983;14:107-22.

[47] Yeomans MR. Palatability and the micro-structure of feeding in humans: the appetizer effect. Appetite 1996;27:119-33.

[48] Davis JD, Smith GP. Analysis of lick rate measure the positive and negative feedback effects of carbohydrates on eating. Appetite 1988;11:229-38. 